\title{
Successful use of an EZ-blocker for lung isolation and management in a hemoptysis patient
}

\author{
Joo Hyung Son, Do Hyung Kim, Sung Kwang Lee \\ Department of Thoracic and Cardiovascular Surgery, Pusan National University Yangsan Hospital, Yangsan, Gyoungnam, South Korea \\ Correspondence to: Sung Kwang Lee, MD. Department of Thoracic and Cardiovascular Surgery, Pusan National University Yangsan Hospital, Pusan \\ National University College of Medicine, 20, Geumo-ro, Mulgeum-eup, Yangsan-si, Gyeongsangnam-do 50612, South Korea. \\ Email: drlsk@naver.com.
}

\begin{abstract}
When massive hemoptysis develops suddenly, patients typically die due to hypovolemia or airway obstruction. Intubation, endobronchial blocking, and elimination of blood clots are urgently required. However, existing double-lumen tubes and single endobronchial balloon systems are inadequate. We herein report successful EZ-blocker-mediated one-lung ventilation of a patient with a massive hemoptysis who required emergency life-saving surgery.
\end{abstract}

Keywords: Hemoptysis; EZ-blocker; lung isolation

Submitted Jul 07, 2019. Accepted for publication Sep 04, 2019.

doi: $10.21037 /$ atm.2019.09.74

View this article at: http://dx.doi.org/10.21037/atm.2019.09.74

When massive hemoptysis develops suddenly, most patients die from hypovolemia or airway obstruction; the airway floods with blood, preventing surgery (1). Rapid endobronchial blocking of the lung bleeding site is essential for airway maintenance and to preserve life. If emergency surgery is required, intubation, endobronchial blocking, and elimination of blood clots from normal lungs should be rapidly and simultaneously performed. However, current double-lumen tubes or single endobronchial balloon systems are inadequate $(2,3)$. We used the EZ-blocker (Teleflex, Raleigh, NC, USA) that has been newly developed for one-lung ventilation of difficult airways in patients with massive hemoptysis who require emergency life-saving surgery (4).

\section{Case presentation}

A 54-year-old male presented with cough, sputum production, and dyspnea 3 days in duration. Chest computed tomography (CT) revealed multifocal consolidations in both lungs, necrotizing abscesses in the right upper and lower lobes, and a suspicion of a pulmonary-esophageal fistula. The patient exhibited respiratory distress and symptoms of septic shock upon admission. After intubation, he underwent mechanical ventilation, and tracheostomy was performed on day 10 of admission. On day 12, a massive hemoptysis developed; blood filled the tracheostomy tube. On bronchoscopy, the hemoptysis filled a right lower lobe volume of about $800 \mathrm{~mL}$. It was difficult to maintain respiration even via application of $100 \%$ oxygen under $14 \mathrm{cmH}_{2} \mathrm{O}$ of pressure and $8 \mathrm{cmH}_{2} \mathrm{O}$ of positive endexpiratory pressure; mechanical ventilation was impossible because of airway obstruction caused by blood and blood clots. We suspected that the tracheostomy site was bleeding, and changed the intubation to orotracheal. However, the tracheostomy site was not bleeding; endotracheal bleeding persisted even after the change to the orotracheal tube. The patient developed hypotension and hypercapnia attributable to hypovolemia and airway obstruction. We decided to perform selective right bronchus blocking and left one-lung ventilation. An EZ-blocker catheter was inserted through the orotracheal intubation tube. The required catheter length was determined by measuring the distance from the tracheostomy site to the carina bifurcation evident on the chest X-ray. After catheter insertion, $5 \mathrm{~mL}$ air was injected into the right-side balloon, aggressive blood suction was performed via the intubation tube, and left ventilation was confirmed using a stethoscope. After repeated blood 
A

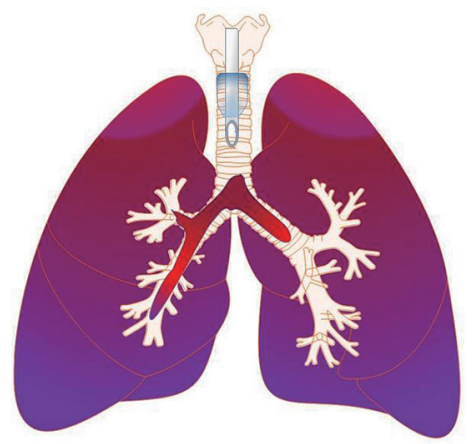

C

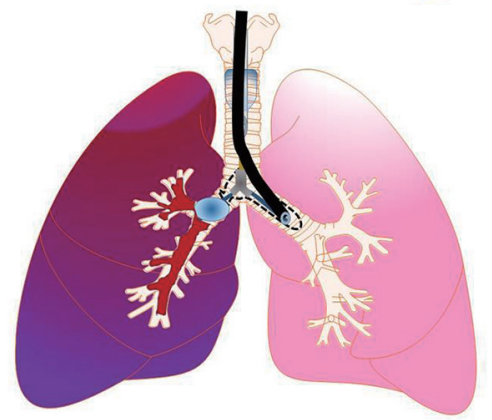

B

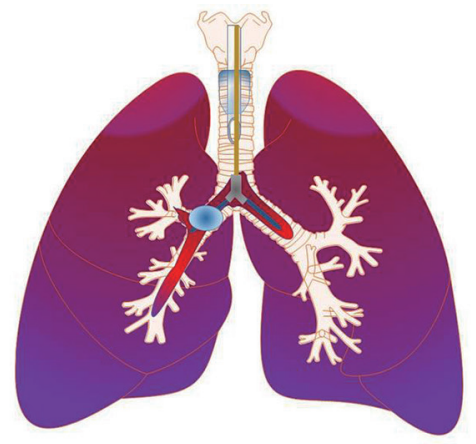

D

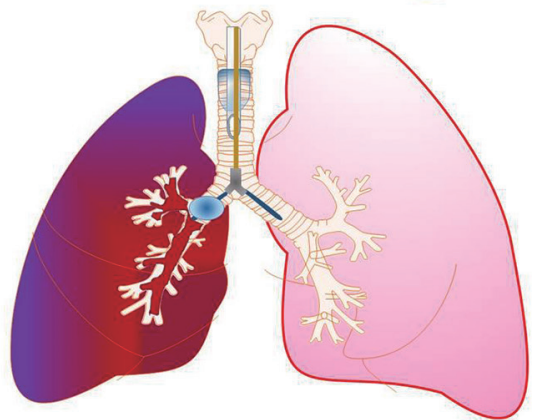

Figure 1 Schematic diagram of EZ-blocker insertion. (A) An endotracheal tube was inserted because blood clots blocked both main bronchi; (B) an EZ-blocker was inserted via the endotracheal tube, and the right-sided balloon was inflated to block the right main bronchus; (C) a bronchoscope was inserted into the left main bronchus to remove blood clots; (D) finally, the left lung was well-ventilated.

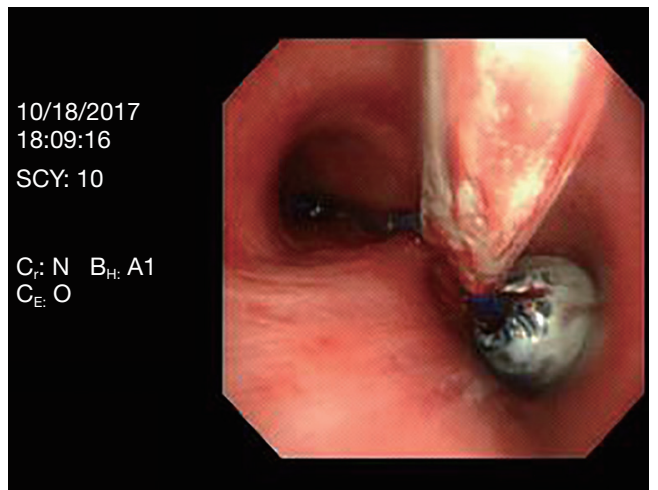

Figure 2 Bronchoscopy revealed that the right main bronchus was completely blocked by the balloon and the left bronchus contained a "de-ballooned" catheter.

suction, the mechanical ventilation and the vital signs stabilized (Figure 1). Bronchoscopy was performed 30 min after endobronchial blocking. Bronchoscopy revealed that the right main bronchus was completely blocked by the balloon and the left bronchus exhibited a de-ballooned catheter (Figure 2). Blood clots were bronchoscopically removed to the level of the peripheral bronchus, and the vital signs maintained via blood transfusion and infusion of inotropic agents. Next, chest CT was performed and revealed that the right main bronchus was blocked by the EZ-blocker balloon. In addition, the cavitary lesion of the right lower lobe exhibited extravasation of the contrast medium, raising a suspicion of a pseudoaneurysm (Figure 3). Chest CT revealed interval aggravation of extensive consolidation in both lungs, aggravation of necrotizing pneumonia combined with aspirated blood, and a suspicion of rupture of a Rasmussen aneurysm. In the mid-esophagus, a fistula between the lung parenchyma and the esophagus was suspected and a new calcified mass was found in the esophagus.

As soon as the patient had stabilized, we performed an emergency operation. As the left lung function was severely compromised by pneumonia and aspiration, veno-venous extracorporeal membrane oxygenation was used to maintain respiratory function during surgery. We performed a right pneumonectomy after one-lung ventilation was established using the EZ-blocker because the right lower and upper lobes had been destroyed by the necrotizing pneumonia. Esophageal cancer accompanied by esophageal perforation was discovered during surgery; we thus performed 

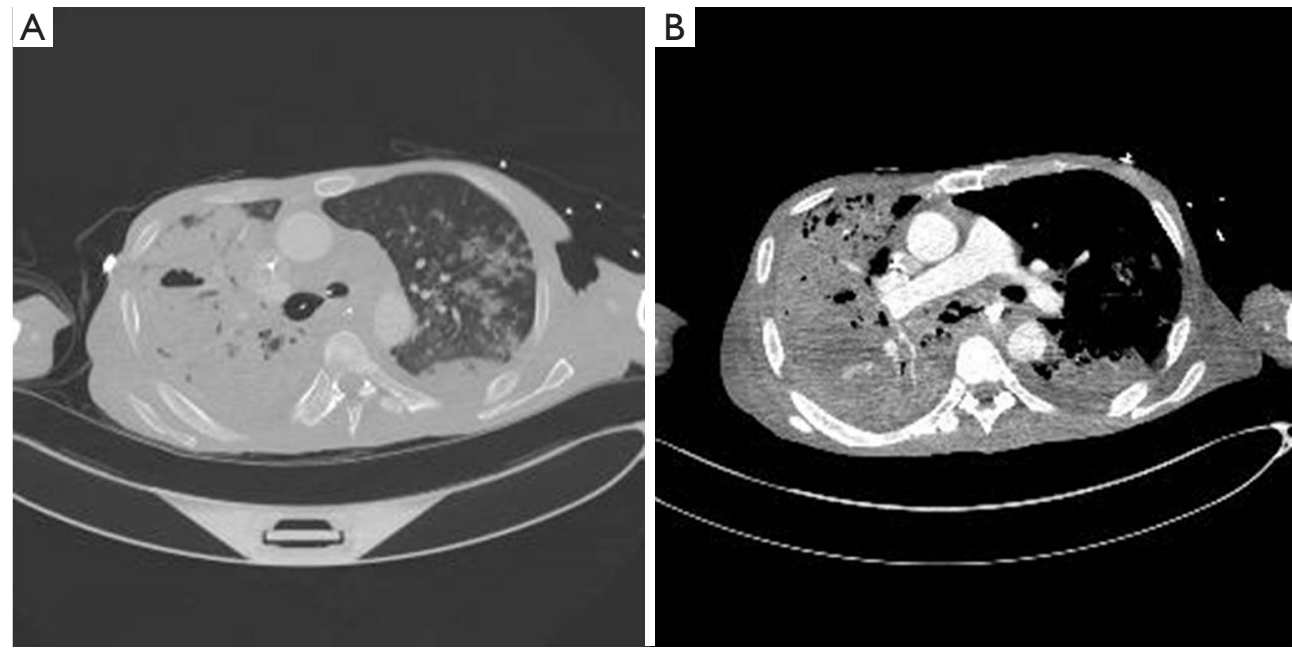

Figure 3 Chest computed tomography (CT) after insertion of EZ blocker. (A) Chest CT revealed that the right main bronchus was blocked by the EZ-blocker balloon; (B) the cavitary lesion of the right lower lobe exhibited extravasation of the contrast medium, raising suspicion of a pseudoaneurysm.

esophagectomy and esophagogastrostomy.

\section{Conclusions}

There are two methods of selective one-side lung blockade. The first blocks the endobronchial lesions with packing material. Although the success rate is high, an expert physician and special instruments such as a rigid bronchoscope are required to perform packing in the context of massive hemoptysis, and the patient must survive until arrival in the operation room; packing cannot be performed in the intensive care unit or the emergency room $(5,6)$. The second (more common) method is selective endobronchial blocking after intubation. An endobronchial balloon blocker or a double-lumen tube is used to prevent aspiration from the bleeding lung. Endobronchial blocking is a hemostatic method with a high success rate [96\%; (7)]. Unlike the first method, endobronchial blocking can be performed in the intensive care unit or the emergency room. However, the method is not rapid; bronchoscopic assistance is always necessary (3). In particular, it is difficult to insert a bronchoscope or a suction catheter (to remove blood clots) through a double-lumen tube because the inner diameter of each lumen is less than that of an endotracheal tube. Although selective blocking is possible using a double-lumen tube, it may be difficult to maintain effective respiration because clot removal is challenging. If, alternatively, an endobronchial balloon blocker is used, it is simple to insert a relatively thick suction catheter or bronchoscope; effective clot removal from a healthy lung is possible if blocking is appropriately performed.

However, it is difficult to precisely place a single endobronchial balloon on a target bronchus. Catheter placement on the left is challenging; the left main bronchus is much more sharply angulated than the right main bronchus. In addition, it is difficult to place the balloon precisely on the right main bronchus because that bronchus is short, and the bronchus of the right upper lobe divides immediately after the right bronchus divides. If the catheter must be deeply placed, adequate endobronchial blocking may be difficult in patients with hemorrhages of the right upper lobe.

As neither of the conventional endobronchial blocking methods allow facile, precise catheter delivery to the target site in the absence of bronchoscopic assistance, endobronchial blocking methods that do not require bronchoscopy are required; bronchoscopy is seldom possible in emergency situations. The EZ-blocker is a new double endobronchial balloon system developed to eliminate the disadvantages of the double lumen tube and the endobronchial blocker; these include possible intubationassociated tracheobronchial injury, challenging intubation in patients with difficult airways, the inconvenience associated with insertion of tubes of different sizes depending on airway diameter, and endobronchial balloon 
blocking by anatomical structures of both bronchi. The EZblocker allows more rapid bilateral balloon placement at the carinal bifurcation site compared to the use of a single endobronchial balloon blocker, and bronchoscopy is not required $(8,9)$. In patients exhibiting massive hemoptysis, endobronchial blocking is achievable; blood flooding has but little effect, and it is then possible to insert a suction catheter that adequately removes clots although the catheter is not of large diameter. In addition, the ability to maintain one-lung ventilation during surgery is invaluable.

In conclusion, successful lung surgery was possible after rapid endobronchial blocking, stable respiratory maintenance, and bleeding control using the EZ-blocker to stem an endobronchial blood flood caused by massive hemoptysis. The EZ-blocker can be easily and rapidly placed compared to the conventional lung isolation method; it can save the lives of patients exhibiting massive hemoptysis and allow safe performance of operations.

\section{Acknowledgments}

None.

\section{Footnote}

Conflicts of Interest: The authors have no conflicts of interest to declare.

Ethical Statement: The authors are accountable for all aspects of the work in ensuring that questions related to the accuracy or integrity of any part of the work are appropriately investigated and resolved. We received written informed consent was obtained from the patient for publication of this case report and any accompanying images to patient.

Cite this article as: Son JH, Kim DH, Lee SK. Successful use of an EZ-blocker for lung isolation and management in a hemoptysis patient. Ann Transl Med 2019;7(22):701. doi: 10.21037/atm.2019.09.74

\section{References}

1. Ficker JH, Bruckl WM, Suc J, et al. Haemoptysis: Intensive care management of pulmonary hemorrhage. Internist (Berl) 2017;58:218-25.

2. Jean-Vivien S, Clément D, Jean-Pierre T, et al. Doublelumen endobronchial tube and alternatives in massive hemoptysis: How do you want to save lives? J Emerg Trauma Shock 2011;4:438.

3. Correia S, Dionisio J, Duro da Costa JJ. Modified technique of endobronchial balloon tamponade for persistent hemoptysis. J Bronchology Interv Pulmonol 2014;21:361-5.

4. Matei A, Tommaso Bizzarri F, Preveggenti V et al. EZBlocker and one-lung ventilation via tracheostomy. J Cardiothorac Vasc Anesth 2015;29:e32-3.

5. Peralta AR, Chawla M, Lee RP. Novel bronchoscopic management of airway bleeding with absorbable gelatin and thrombin slurry. J Bronchology Interv Pulmonol 2018;25:204-11.

6. Valipour A, Kreuzer A, Koller H, et al. Bronchoscopyguided topical hemostatic tamponade therapy for the management of life-threatening hemoptysis. Chest 2005;127:2113-8.

7. Freitag L, Tekolf E, Stamatis G, et al. Three years experience with a new balloon catheter for the management of haemoptysis. Eur Respir J 1994;7:2033-7.

8. Moritz A, Irouschek A, Birkholz T, et al. The EZ-blocker for one-lung ventilation in patients undergoing thoracic surgery: Clinical applications and experience in 100 cases in a routine clinical setting. J Cardiothorac Surg 2018;13:77.

9. Templeton TW, Templeton LB, Lawrence AE, et al. An initial experience with an extraluminal EZ-Blocker(R): A new alternative for 1-lung ventilation in pediatric patients. Paediatr Anaesth 2018;28:347-51. 